\title{
A novel homozygous mutation in the glycerol-3-phosphate dehydrogenase 1 gene in a Chinese patient with transient infantile hypertriglyceridemia: a case report
}

\author{
Jia-Qi Li ${ }^{1}$, Xin-Bao Xie ${ }^{2 *}$, Jia-Yan Feng ${ }^{3}$, Lian Chen ${ }^{3}$, Kuerbanjiang Abuduxikuer ${ }^{2}$, Yi Lu ${ }^{2}$, Yu-Chuan Li ${ }^{2}$ \\ and Jian-She Wang ${ }^{2^{*}}$
}

\begin{abstract}
Background: Transient infantile hypertriglyceridemia (HTGTI) is an autosomal recessive disorder caused by mutations in the glycerol-3-phosphate dehydrogenase 1 (GPD1) gene.

Case presentation: We report a case of HTGTI in a Chinese female infant. She presented with hepatomegaly, hypertriglyceridemia, moderately elevated transaminases, and hepatic steatosis at 3.5 months of age. A novel mutation c.523C>T, p. (Q175*) was identified in GPD1. The patient was a homozygote and her parents were heterozygous for the mutation. Ultrastructural study showed intrahepatocytic lipid droplets.

Conclusions: This is the first reported case of HTGTI in Chinese, expanding the worldwide distribution of HTGTI and the mutation spectrum of GPD1.
\end{abstract}

Keywords: Hypertriglyceridemia, GPD1, hepatomegaly, hepatic steatosis, HTGTI

\section{Background}

The glycerol-3-phosphate dehydrogenase 1 (GPD1; MIM 138420) gene, which is mapped to chromosome 12q12-q13, encodes cytoplasmic NAD-dependent GPD1 that is crucial in both carbohydrate and lipid metabolism by catalyzing the reversible redox reaction of dihydroxyacetone phosphate (DHAP) and reduced nicotine adenine dinucleotide (NADH) to glycerol-3-phosphate (G3P) and $\mathrm{NAD}^{+}[1,2]$. Under physiological conditions, the reaction strongly favors the formation of G3P [2]. Glycerol-3-phosphate dehydrogenase 2 (GPD2; MIM 138430) is located on the outer surface of the inner mitochondrial membrane and catalyzes the unidirectional reaction of G3P and flavin adenine dinucleotide (FAD) to DHAP and $\mathrm{FADH}_{2}$ [2]. Together with a mitochondrial GPD2, GPD1 forms the G3P shuttle mainly in the brain and skeletal muscle of mammals, which

\footnotetext{
* Correspondence: xxb116@163.com; jshwang@shmu.edu.cn

${ }^{2}$ The Center for Pediatric Liver Disease, Children's Hospital of Fudan

University, 399 Wanyuan Road, Minhang District, Shanghai 201102, China

Full list of author information is available at the end of the article
}

transfers reducing equivalents from the cytosol to the mitochondria [3]. The human GPD1 is organized into two distinct domains, the $N$-terminal eight-stranded $\beta$-sheet sandwich domain (from residues 3-190) and the $C$-terminal helical substrate-binding (from residues 193349) domain [2]. NAD ${ }^{+}$binds to GPD1 at the periphery of its $\beta$-sheet core (10-GSGNWG-15) [2].

GPD1 mutations were first identified by Basel-Vanagaite et al. as the cause of transient infantile hypertriglyceridemia (HTGTI; OMIM 614480) in 10 individuals from four consanguineous Israeli Arab families carrying a homozygous founder mutation c.361-1G>C [3]. HTGTI manifests as early onset hepatomegaly, hypertriglyceridemia, moderately elevated transaminases, hepatic steatosis, and hepatic fibrosis [3]. Recently, Li et al. reported biallelic mutations in GPD1 gene of a Chinese boy who presented with a different phenotype comprising obesity, insulin resistance, fatty liver, and short stature [4]. To date, only four reports have described 16 patients harboring homozygous or compound heterozygous mutations in the GPD1 gene [3-6]. Here, we describe a Han Chinese

(c) The Author(s). 2018 Open Access This article is distributed under the terms of the Creative Commons Attribution 4.0 International License (http://creativecommons.org/licenses/by/4.0/) which permits unrestricted use, distribution, and reproduction in any medium, provided you give appropriate credit to the original author(s) and the source, provide a link to the Creative Commons license, and indicate if changes were made. The Creative Commons Public Domain Dedication waiver (http://creativecommons.org/publicdomain/zero/1.0/) applies to the data made available in this article, unless otherwise stated. 
patient with a novel GPD1 homozygous mutation who presented with hepatomegaly, elevated transaminases, hypertriglyceridemia, and fatty liver in infancy.

\section{Case presentation}

This study was approved by the Research Ethics Committee of Children's Hospital of Fudan University and was conducted under the Declaration of Helsinki ethical principles for medical research involving human subjects. Informed consent was obtained from the child's parents.

\section{Patient}

The female patient was the second child of a non-consanguineous Han Chinese couple. The mother previously had four episodes of spontaneous abortion during the first trimester due to lack of mixed lymphocyte reaction blocking antibodies. Through paternal lymphocyte immunotherapy, the mother successfully gave birth to the proband and her older brother. Fetal ultrasound scans of the proband showed fetal liver was normal. She was born at the gestational age of 39 weeks by cesarean section, with a birth weight of $3.15 \mathrm{~kg}$. She presented at the age of 3 months and 14 days with bronchopneumonia, during the course of which elevated alanine aminotransferase (101 U/L, normal: 0-40 U/L) and aspartate aminotransferase (135 U/L, normal: 0-40 U/L) were noted. Initial abdominal ultrasound revealed hepatomegaly (the liver enlarged $2 \mathrm{~cm}$ below the costal margin). As the elevated liver enzymes and hepatomegaly persisted after recovery of bronchopneumonia, the child was transferred to another hospital for further treatment. Fasting hypertriglyceridemia was noted at presentation and throughout the follow-up period (range 4.39-10.94 mmol/L, normal: 0.56-1.7 $\mathrm{mmol} / \mathrm{L}$ ). Gamma-glutamyltranspeptidase and total bile acids were $265 \mathrm{IU} / \mathrm{L}$ (normal: 7-50 IU/L) and $18.2 \mu \mathrm{mol} / \mathrm{L}$ (normal: $0-10 \mu \mathrm{mol} / \mathrm{L}$ ), respectively. However, total cholesterol, lipoprotein, bilirubin, alpha-fetoprotein and synthetic liver function (coagulation studies and albumin) were within normal limits. Abdominal CT scan showed a severely enlarged liver accompanied with fatty change, which had diffusely decreased density. Viral serological markers (hepatotrophic viruses, Epstein-Barr virus, cytomegalovirus, and HIV), toxoplasma, thyroid function tests, creatine phosphokinase, glucose, ceruloplasmin, blood routine tests, urine routine tests, blood lactate, blood ammonia, carbonyldiamide, creatinine, uric acid, urine organic acids, autoimmune serology, immunoglobulin levels, and echocardiogram were unremarkable. At this point, due to the combination of hypertriglyceridemia, hepatomegaly, and elevated liver transaminases, the working diagnosis was inclined to inherited metabolic liver disease. Thus, the whole blood samples from the family were sent to Precision MD (Beijing, China) for panel sequencing to screen 4503 genes using the customized xGen Inherited Diseases Panel. She took "Compound Glycyrrhizin" during this hospitalization. However, the levels of triglyceride and liver enzymes were not decreased. She was referred to the center for pediatric liver diseases in our hospital at 6.5 months of age for percutaneous liver biopsy. Since then, she did not receive any treatment. At the last evaluation at the age of 1 year and 3 months, the child still presented with hepatomegaly, hypertriglyceridemia, and moderately elevated transaminases, while maintaining a normal growth and psychomotor development. The detailed clinical characteristics of the proband are shown in Table 1. The patient's parents and older brother were asymptomatic. The liver functions of the mother and older brother were normal. However, alanine aminotransferase $(59 \mathrm{IU} / \mathrm{L}$, normal: 0-40 IU/L), gamma-glutamyl transpeptidase $(62$ IU/L, normal: 7-50 IU/L), total cholesterol (5.87 $\mathrm{mmol} / \mathrm{L}$, normal: $3.1-5.2 \mathrm{mmol} / \mathrm{L}$ ) and triglyceride (3.5 mmol/L, normal: $0.56-1.7 \mathrm{mmol} / \mathrm{L}$ ) were mildly elevated in the father. In addition, the father was morbidly obese (body mass index $31.3 \mathrm{~kg} / \mathrm{m}^{2}$, normal: $18.5-23.9 \mathrm{~kg} / \mathrm{m}^{2}$ ).

\section{Genetic analysis}

Written informed consent was obtained prior to the collection of peripheral blood from the proband and her family members. All the blood samples were sent to Precision MD (Beijing, China) for sequencing. Genomic DNA of all the family members was extracted from their peripheral blood leukocytes according to the manufacturer's standard procedure using the QIAamp DNA Blood Midi Kit (Qiagen, Hilden, Germany). Genomic DNA of the proband underwent panel sequencing to screen 4503 genes using the customized xGen Inherited Diseases Panel. DNA was fragmented by Covaris LE220 (Massachusetts, USA) to generate paired-end library (200-250 bp). Targets were captured using xGen Lockdown Probes (Precision MD, Beijing, China). The captured libraries were sequenced using the Illumina hiseq2500 Analyzers following the manufacturer's instructions (Illumina, San Diego, USA). The reads were mapped to human genome reference (hg19) using Burrows-Wheeler Aligner (http://bio-bwa.sourceforge.net/). Single-nucleotide variants and indels were called with SOAPsnp (The Beijing Genomics Institute in-house software) and Samtools (http://samtools.sourceforge.net/). All single-nucleotide variants and indels were filtered via multiple databases. Pathogenic variants were assessed under the protocol issued by the American College of Medical Genetics and Genomics 
Table 1 Clinical and laboratory findings of the patient

\begin{tabular}{|c|c|}
\hline \multicolumn{2}{|l|}{ Personal history } \\
\hline Presenting age (months) & 3.5 \\
\hline Age at last following up (months) & 15 \\
\hline Gravidity $(\mathrm{G})$ and Parity $(\mathrm{P})$ & G6P2 \\
\hline Gestation (weeks) & 39 \\
\hline Birth weight (g) & 3150 \\
\hline Birth history & cesarean section \\
\hline \multicolumn{2}{|l|}{ Physical examination at 6.5 months old } \\
\hline Head circumference (cm) & 42.0 \\
\hline Chest circumference $(\mathrm{cm})$ & 43.0 \\
\hline Height (cm) & 63.0 \\
\hline Weight (kg) & 6.8 \\
\hline Temperature $\left({ }^{\circ} \mathrm{C}\right)$ & 37.0 \\
\hline Heart rate (beats per minute) & 126 \\
\hline Respiration rate (times per minute) & 28 \\
\hline Hepatomegaly & Yes \\
\hline Splenomegaly & No \\
\hline \multicolumn{2}{|l|}{ Biochemical examination } \\
\hline Albumin (g/L) & $45.1-50.8$ \\
\hline Alanine aminotransferase (IU/L) & $68-110$ \\
\hline Aspartate aminotransferase (IU/L) & 108-186 \\
\hline Gammaglutamyl-transpeptidase (IU/L) & $233-482$ \\
\hline Direct bilirubin (umol/L) & $1.0-2.3$ \\
\hline Total bilirubin (umol/L) & 2.4-9.4 \\
\hline Total bile acid (umol/L) & $9.0-19.8$ \\
\hline Alpha fetal protein $(\mathrm{ng} / \mathrm{mL})$ & 53 \\
\hline Triglyceride (mmol/L) & 4.18-10.94 \\
\hline Total cholesterol (mmol/L) & $2.36-4.35$ \\
\hline Glucose (mmol/L) & $4.2-5.7$ \\
\hline \multicolumn{2}{|l|}{ Coagulation test } \\
\hline D-dimer (mg/L) & $0.27-1.18$ \\
\hline Activated partial thromboplastin time (s) & $25.0-33.3$ \\
\hline Thrombin time (s) & 19.4-19.9 \\
\hline Prothrombin time (s) & $11.0-13.3$ \\
\hline Fibrinogen ( $g / L)$ & $1.84-1.93$ \\
\hline \multicolumn{2}{|l|}{ Blood routine examination } \\
\hline Hemoglobin (g/L) & $95.0-101.0$ \\
\hline Red blood cell count (per liter) & $4.91-5.24 \times 10^{12}$ \\
\hline White blood cell count (per liter) & $7.7-11 \times 10^{9}$ \\
\hline Lymphocytes (\%) & $65.0-75.0$ \\
\hline Neutrophils (\%) & $16.8-22.0$ \\
\hline Platelets count (per liter) & $568-782 \times 10^{9}$ \\
\hline
\end{tabular}

[7]. Mutations were verified in the proband and her family members by Sanger sequencing.

\section{Molecular findings}

A homozygous nonsense mutation c.523C $>\mathrm{T}$, p. (Q175*) in GPD1 (NM_005276.3) was identified in the proband. Her parents and older brother were heterozygous for the mutation, consistent with a recessive inheritance (Additional file 1). This variant introduces a stop codon at codon 175, which is predicted to result in nonsense mediated mRNA decay by MutationTaster (http:// www.mutationtaster.org/) [8]. The variant was novel and not recorded in Exome Aggregation Consortium Server (http://exac.broadinstitute.org/), NHLBI Exome Sequencing Project (http://evs.gs.washington.edu/EVS/) and Thousand Genomes Project (http://www.1000genomes.org/home).

\section{Pathological findings}

Liver biopsy was performed at 6.5 months of age. Light microscopy revealed marked micro- and macro- vacuoles in hepatocytes on hematoxylin and eosin stained sections, indicating severe hepatic steatosis (Fig. 1a). Mild lymphocytic inflammatory infiltrates in the portal tracts were also present. Periodic acid Schiff staining showed macro- and micro- vacuoles in hepatocytes (Fig. 1b). Masson staining revealed mild portal fibrosis (Fig. 1c). Immunostaining for cytokeratin 7 and 19 demonstrated slight proliferation of the bile ducts along the ductal plate. Iron, $\mathrm{HBsAg}, \mathrm{HBcAg}$ and Epstein-Barr virus were negative. Electron microscopy revealed macro- and micro fat and glycogen in the cytoplasm of hepatocytes, indicating severe liver steatosis (Fig. 1d). Increased myeloid body, abundant collagen fibers and mild dilation of bile canaliculi were also observed.

\section{Discussion and conclusions}

HTGTI is a rare autosomal recessive disorder that has been described in a total of 15 affected individuals in three studies $[3,5,6]$. The shared features of these patients include infantile hypertriglyceridemia, elevated liver enzymes, hepatomegaly, liver steatosis and fibrosis. Additional uncommon phenotypes include fasting hypoglycemia and kidney disease. We report the first Han Chinese patient with HTGTI, and the patient carried a novel homozygous nonsense mutation in the GPD1 gene, which is the causative variation. Our patient clinically resembled other reported children with HTGTI: She presented with elevated liver transaminases, hypertriglyceridemia, marked hepatomegaly, and hepatic steatosis since early infancy. Electron microscopy was only undertaken in one of 15 previous affected patients, revealing interhepatocytic, not intrahepatocytic, vesicles 


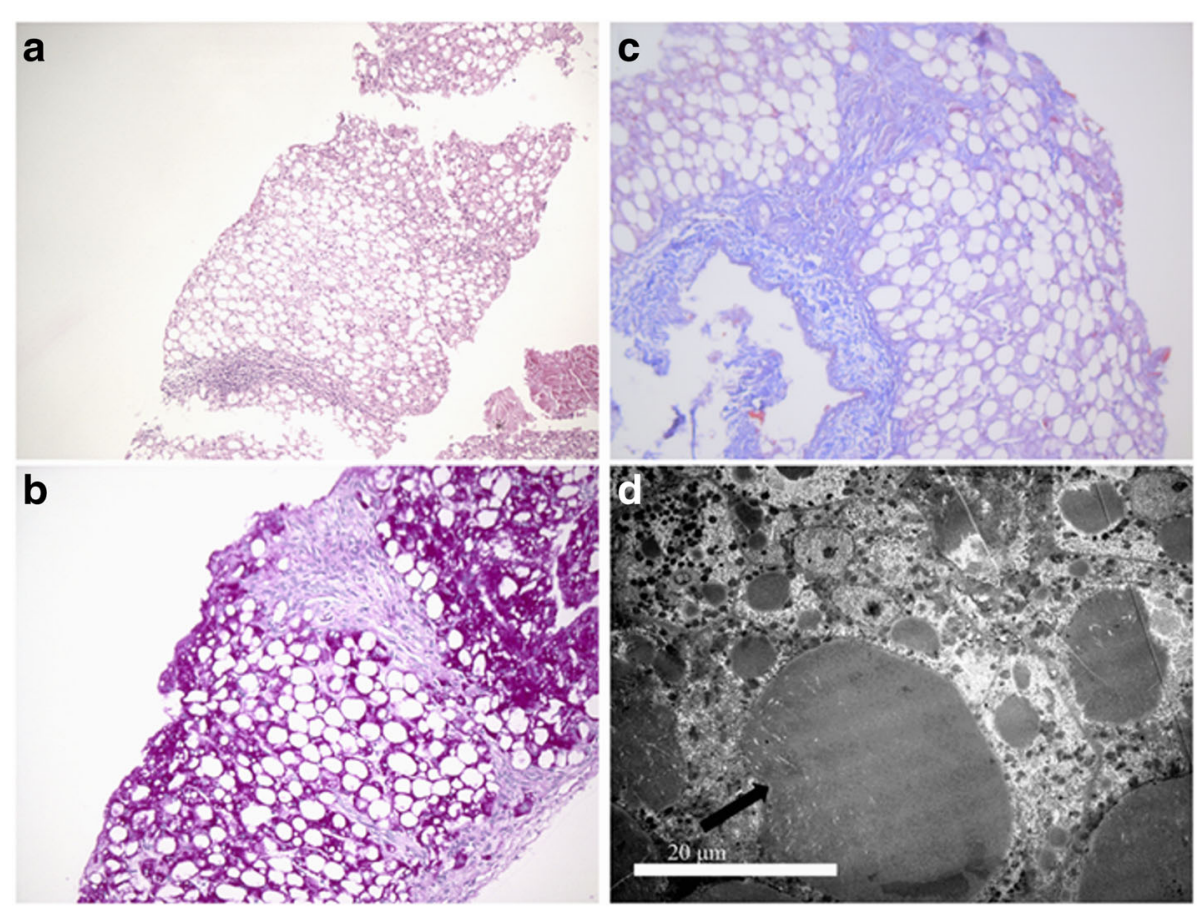

Fig. 1 The pathological results of the proband. a Marked macro- and microvacuoles were observed (Hematoxylin and Eosin staining; original magnification, $\times 100)$. b Periodic acid Schiff staining also showed macro- and microvacuoles in hepatocytes ( $\times 200)$. c Mild fibrosis was presented in portal tracts (Masson staining, $\times 200$ ). $\mathbf{d}$ The arrow showed fats were in the hepatocytes

(most likely fat) [6]. By contrast, electron microscopy showed intrahepatocytic, not interhepatocytic, lipid droplets in our patient; however, the significance of this difference is unclear. Although our patient was not given treatment since 6.5 months old, the levels of triglyceride and liver enzymes decreased at the last evaluation at the age of 1 year and 3 months. The heterozygote (carrier) parents and older brother of our reported patient were asymptomatic. The mildly elevated liver functions of the father may be due to his high body mass index.

Recently, Li et al. reported biallelic mutations (c.220-2A > G and c.820G>A) in GPD1 gene of a 13-year, 8-month-old Chinese boy who presented with obesity, insulin resistance, fatty liver, dermal abnormalities (facial acne, acanthosis nigricans, and hirsutism), short stature, elevated dehydroepiandrosterone sulfate and lipoprotein- $\alpha$ levels, which were different from HTGTI phenotype [4]. Clinical features and molecular genetics of individuals with biallelic mutations in GPD1 gene were shown in Table 2. Li et al. also suggested that the GPD1 gene should be considered as the short stature causing gene [4]. However, it is uncertain that the different phenotype of the adolescent patient was due to phenotypic heterogeneity or having an unrecognized second disorder.

Hypertriglyceridemia is a hallmark of many disorders, including metabolic syndrome, diabetes, atherosclerosis, and obesity. Several genetic defects for hypertriglyceridemia have been identified, including mutations in APOA5 (MIM 606368) [9], LIPI (MIM 609252) [10], LPL (MIM 609708) [11], APOC2 (MIM 608083) [12], LIPC (MIM 151670) [13], USF1 (MIM 191523) [14], GPIHBP1 (MIM 612757) [15], and LMF1 (MIM 611761) [16]. GPD1 disease is one of the important molecular etiologies of primary hypertriglyceridemia with onset in infancy [3]. Basel-Vanagaite et al. confirmed that mutation of GPD1 in HepG2 cells causes increased triglyceride synthesis and secretion [3]. However, the exact mechanism of hypertriglyceridemia in GPD1 deficiency is unclear and remains to be further clarified. Notably, high triglyceride level is an independent risk factor for coronary artery disease and also associated with an increased risk of acute pancreatitis [17-19]. The long term effects of hypertriglyceridemia on patients with HTGTI is unknown, and it is necessary to pay attention to these patients in their long-term follow up.

All the GPD1 deficiency patients had fatty liver on ultrasound or other imaging examinations. Light microscopy undertaken in 6 of sixteen previous affected patients showed steatosis with varying degree of fibrosis. Joshi $\mathrm{M}$ et al. proposed that fatty liver in GPD1 disease may be due to acylation of excess DHAP [5]. The detailed mechanisms are not clear and further research is needed to shed light on them. Fatty liver with 


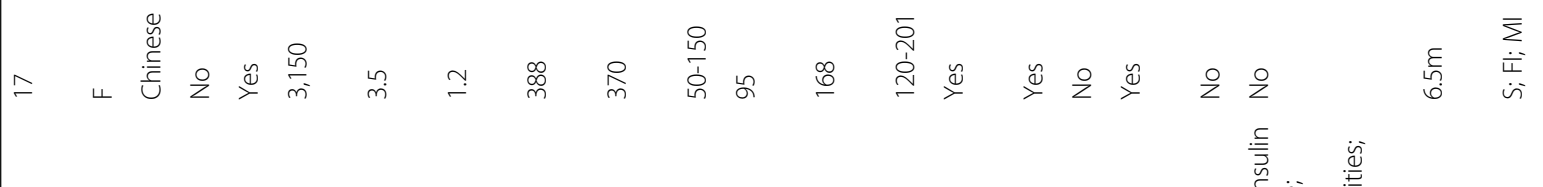

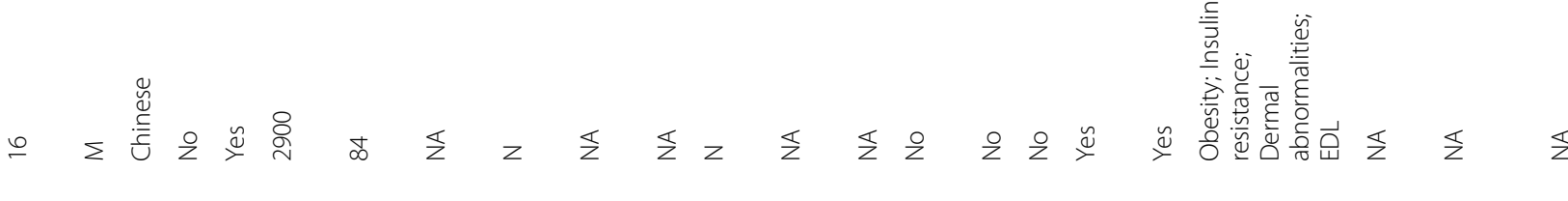

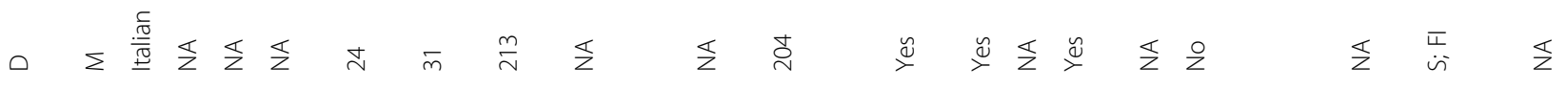

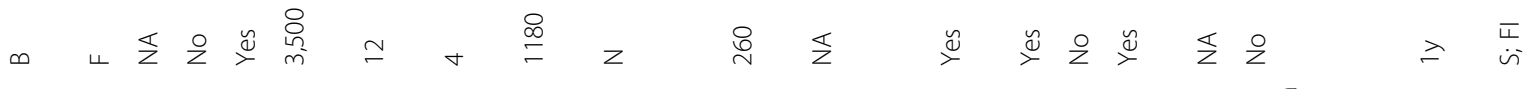

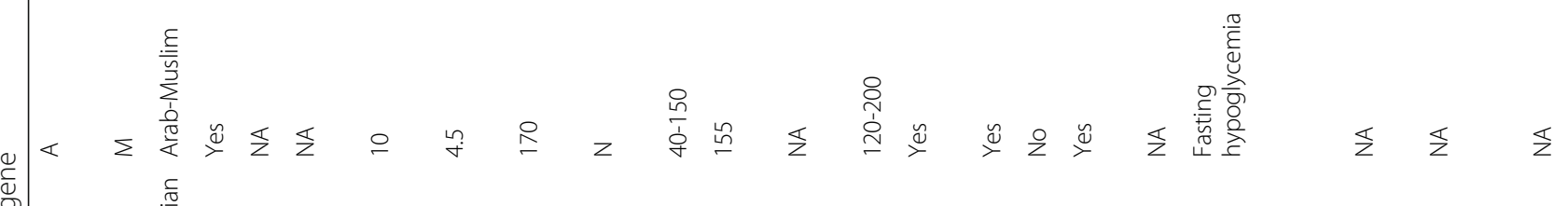

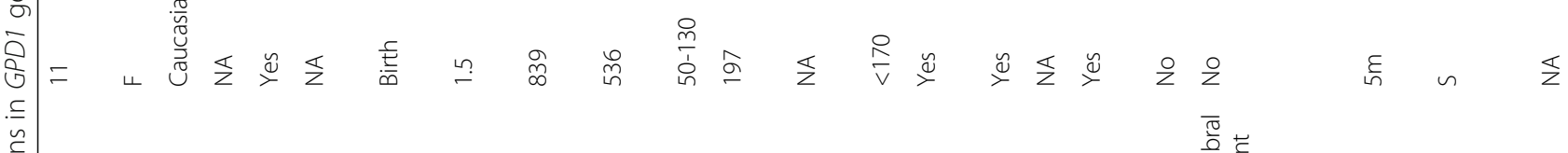

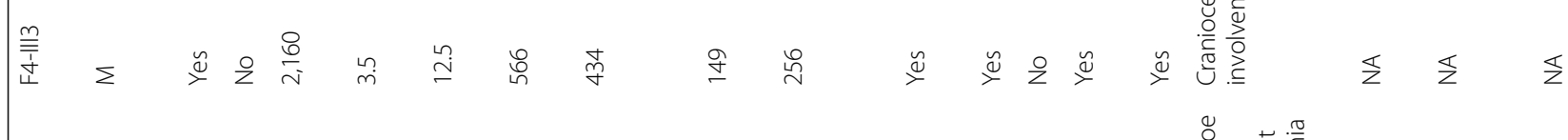

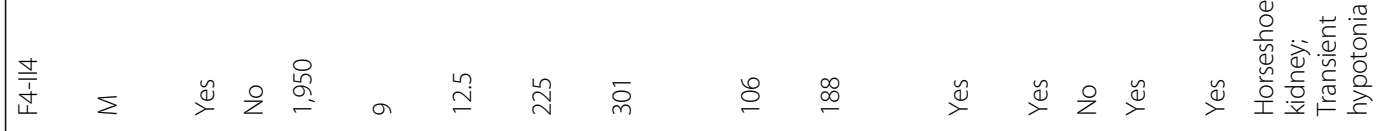

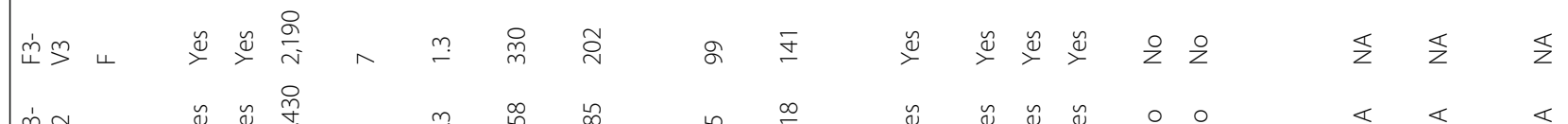

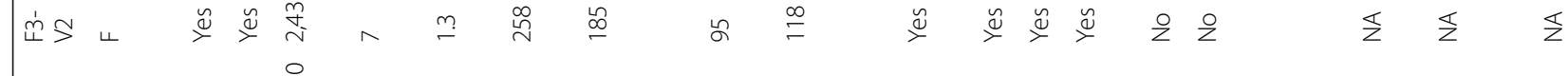

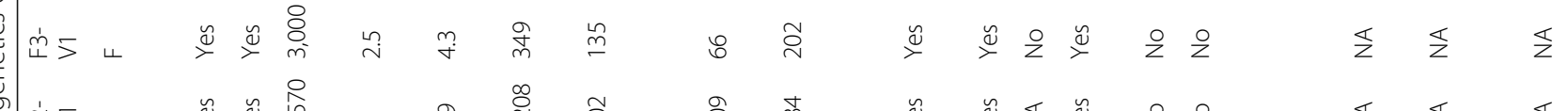

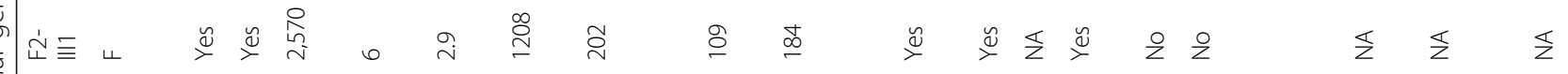

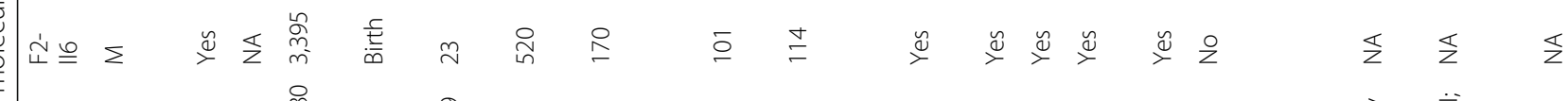

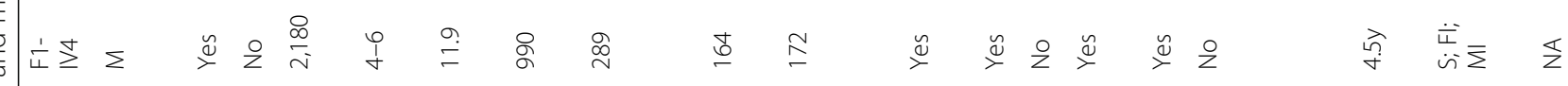

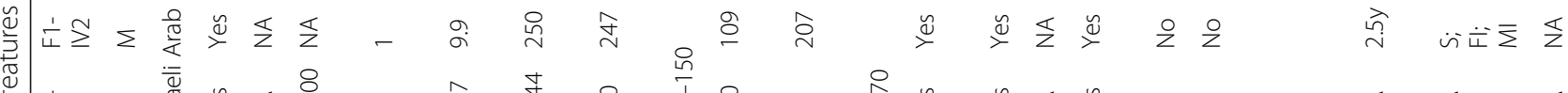

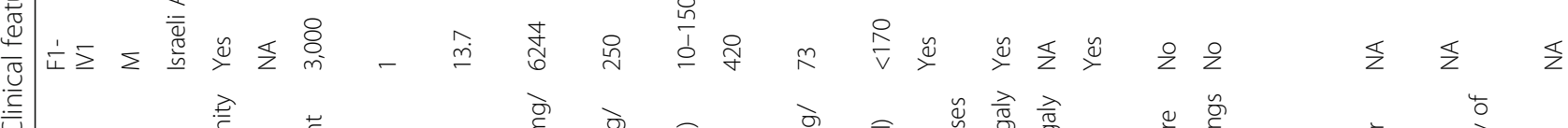

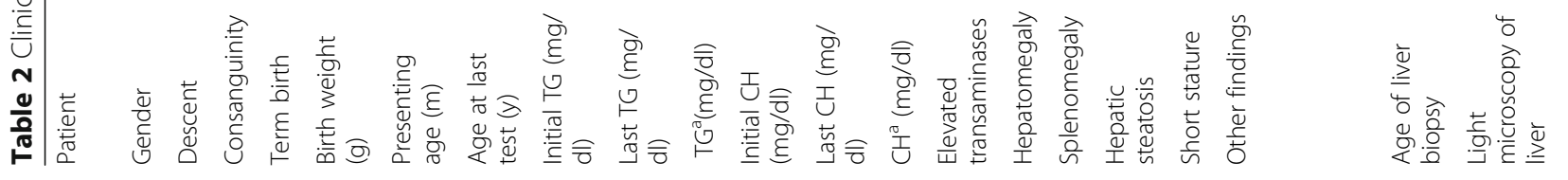




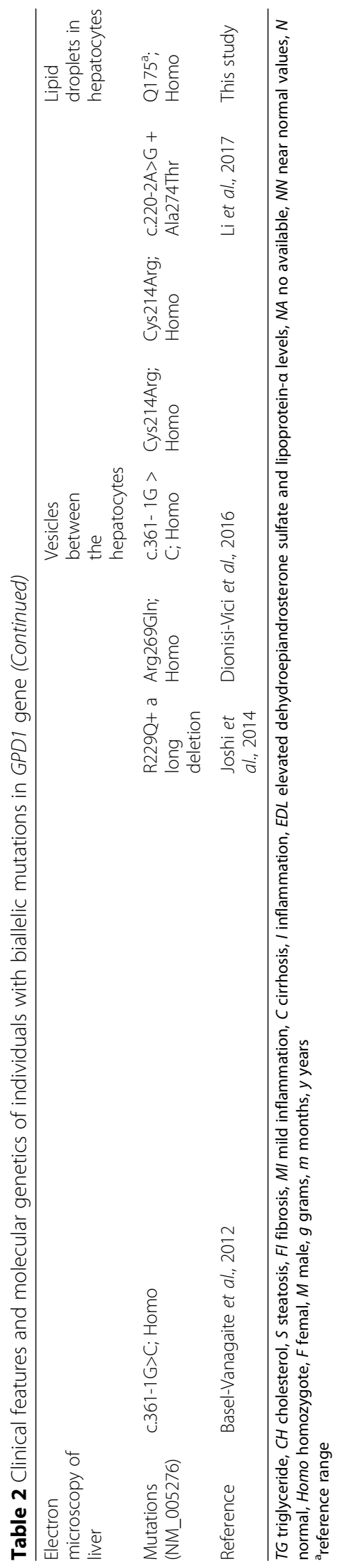


hepatomegaly can be caused by a spectrum of inherited metabolic liver diseases, such as glycogen storage disease, lipidosis, lysosomal diseases, and citrin deficiency. Liver biopsy and genetic analysis are both useful for differential diagnosis of HTGTI and those diseases. However, the definite diagnosis of HTGTI depends on genetic testing.

The natural history of HTGTI is not clear yet. Although most patients presented with liver fibrosis in early infancy, all affected individuals had a good prognosis. At the time of their last evaluation, the levels of triglyceride and liver enzymes were improved in most patients. In addition, the oldest patient (aged 31 years) was doing well [6]. Thus, liver transplantation is not recommended for HTGTI patients. Since triglyceride levels could be improved without specific therapy, we don't recommend lipoprotein apheresis as routine therapy for these patients. But it could be an option for patients with severe hyperlipemia. We recommend to evaluate the growth and development and test liver function, total cholesterol, triglycerides, abdominal ultrasound, FibroScan and other abnormal index in the follow-up visit. In addition, it is necessary to pay attention to coronary artery disease and pancreatitis in their long-term follow up, which is associated with hypertriglyceridemia.

Previously, a total of 5 mutations in the GPD1 gene have been identified in 15 patients with HTGTI. In this study, we reported a novel disease-causing mutation c. $523 \mathrm{C}>\mathrm{T}$, which is predicted to result in nonsense mediated mRNA decay. Among the 16 patients carrying biallelic mutations in GPD1, 15 patients showed a homozygous mutation, while only 1 patient was compound heterozygotes. The identified variants comprised 1 nonsense (c.523C $>$ T), 3 missense (c.686G $>A, \quad$ c.806G $>A$, and c. $640 \mathrm{~T}>\mathrm{C}), 1$ splice site variant (c.361-1G>C), and 1 deletion mutation $(28.7 \mathrm{~kb}>$ the deletion size $>1.85 \mathrm{~kb})$.

In conclusion, we reported a Han Chinese HTGTI patient with mutation of GPD1, having hypertriglyceridemia, hepatomegaly, mildly elevated transaminases, and hepatic steatosis. GPD1 deficiency should also be considered in children and adolescents with hypertriglyceridemia and hepatic steatosis of unknown causes, and that the natural history of the condition is still unknown.

\section{Additional file}

Additional file 1: Mutation analysis of the GPD1 gene in the proband and her parents. The patient was a homozygote and her parents were heterozygous for the mutation. (DOCX $853 \mathrm{~kb}$ )

\section{Abbreviations}

DHAP: Dihydroxyacetone phosphate; FAD: Flavin adenine dinucleotide; G3P: Glycerol-3-phosphate; GPD1: Glycerol-3-phosphate dehydrogenase 1;
GPD2: Glycerol-3-phosphate dehydrogenase 2; HTGTI: Transient infantile hypertriglyceridemia; NADH: Reduced nicotine adenine dinucleotide

\section{Acknowledgments}

The authors are grateful for the support of the family of the patient whom we have studied, and thank referring physicians, nurses, and technical staff.

\section{Funding}

This research was supported by the National Natural Science Foundation of China, Grant Numbers 81570468; Shanghai Medical Key Specialty

Construction Project Number ZK2015A04.

Availability of data and materials

All data generated or analysed during this study are included in this published article (and its Additional files).

\section{Authors' contributions}

JSW, XBX and JQL drafted the manuscript; JSW, XBX, JQL, KA were involved in the acquisition, analysis and interpretation of clinical and genetic data; $Y L$ and YCL were involved in the acquisition, analysis and interpretation of clinical data; JYF and LC performed analysis and interpretation of pathologic data; All authors read and approved the final manuscript.

\section{Ethics approval and consent to participate}

This study was approved by the Research Ethics Committee of Children's Hospital of Fudan University and was conducted under the Declaration of Helsinki ethical principles for medical research involving human subjects. Informed consent was obtained from the child's parents.

\section{Consent for publication}

Written informed consent for publication of clinical details was obtained from the guardians of the patient.

\section{Competing interests}

The authors declare that they have no competing interests.

\section{Publisher's Note}

Springer Nature remains neutral with regard to jurisdictional claims in published maps and institutional affiliations.

\section{Author details}

${ }^{1}$ Department of Pediatrics, Jinshan Hospital of Fudan University, 1508 Longhang Road, Jinshan District, Shanghai 201508, China. ${ }^{2}$ The Center for Pediatric Liver Disease, Children's Hospital of Fudan University, 399 Wanyuan Road, Minhang District, Shanghai 201102, China. ${ }^{3}$ Department of Pathology, Children's Hospital of Fudan University, 399 Wanyuan Road, Minhang District, Shanghai 201102, China.

Received: 10 February 2018 Accepted: 19 June 2018

Published online: 25 June 2018

\section{References}

1. Menaya J, Gonzalez-Manchon C, Parrilla R, Ayuso MS. Molecular cloning, sequencing and expression of a CDNA encoding a human liver NADdependent alpha-glycerol-3-phosphate dehydrogenase. Biochim Biophys Acta. 1995;1262:91-4.

2. Ou X, Ji C, Han X, Zhao X, Li X, Mao Y, et al. Crystal structures of human glycerol 3-phosphate dehydrogenase 1 (GPD1). J Mol Biol. 2006;357:858-69.

3. Basel-Vanagaite L, Zevit N, Zahav AH, Guo L, Parathath S, Pasmanik-Chor M, et al. Transient infantile hypertriglyceridemia, fatty Liver, and hepatic fibrosis caused by mutated GPD1, encoding glycerol-3-phosphate dehydrogenase 1 . Am J Hum Genet. 2012;90:49-60.

4. Li N, Chang G, Xu Y, Ding Y, Li G, Yu T, et al. Biallelic mutations in GPD1 gene in a Chinese boy mainly presented with obesity, insulin resistance, fatty liver, and short stature. Am J Med Genet A. 2017;173:3189-94.

5. Joshi M, Eagan J, Desai NK, Newton SA, Towne MC, Marinakis NS, et al. A compound heterozygous mutation in GPD1 causes hepatomegaly, steatohepatitis, and hypertriglyceridemia. Eur J Hum Genet. 2014;22: $1229-32$. 
6. Dionisi-Vici C, Shteyer E, Niceta M, Rizzo C, Pode-Shakked B, Chillemi G, et al. Expanding the molecular diversity and phenotypic spectrum of glycerol 3phosphate dehydrogenase 1 deficiency. J Inherit Metab Dis. 2016;39:689-95.

7. Richards S, Aziz N, Bale S, Bick D, Das S, Gastier-Foster J, et al. Standards and guidelines for the interpretation of sequence variants: a joint consensus recommendation of the American College of Medical Genetics and Genomics and the Association for Molecular Pathology. Genet Med. 2015; 17:405-24.

8. Schwarz JM, Cooper DN, Schuelke M, Seelow D. MutationTaster2: mutation prediction for the deep-sequencing age. Nat Methods. 2014;11:361-2.

9. Kao JT, Wen HC, Chien KL, Hsu HC, Lin SW. A novel genetic variant in the apolipoprotein A5 gene is associated with hypertriglyceridemia. Hum Mol Genet. 2003;12:2533-9.

10. Wen XY, Hegele RA, Wang J, Wang DY, Cheung J, Wilson M, et al. Identification of a novel lipase gene mutated in Ipd mice with hypertriglyceridemia and associated with dyslipidemia in humans. Hum Mol Genet. 2003;12:1131-43.

11. Henderson HE, Ma Y, Hassan MF, Monsalve MV, Marais AD, Winkler F, et al. Amino acid substitution (lle194_- Thr) in exon 5 of the lipoprotein lipase gene causes lipoprotein lipase deficiency in three unrelated probands. Support for a multicentric origin. J Clin Invest. 1991;87:2005-11.

12. Baggio G, Manzato E, Gabelli C, Fellin R, Martini S, Enzi GB, et al. Apolipoprotein C-II deficiency syndrome. Clinical features, lipoprotein characterization, lipase activity, and correction of hypertriglyceridemia after apolipoprotein C-II administration in two affected patients. J Clin Invest. 1986;77:520-7.

13. Hegele RA, Vezina C, Moorjani S, Lupien PJ, Gagne C, Brun LD, et al. A hepatic lipase gene mutation associated with heritable lipolytic deficiency. Clin Endocrinol Metab. 1991;72:730-2.

14. Pajukanta P, Lilja HE, Sinsheimer JS, Cantor RM, Lusis AJ, Gentile M, et al. Familial combined hyperlipidemia is associated with upstream transcription factor 1 (USF1). Nat Genet. 2004;36:371-6.

15. Wang J, Hegele RA. Homozygous missense mutation (G56R) in glycosylphosphatidylinositol-anchored high-density lipoprotein-binding protein 1 (GPI-HBP1) in two siblings with fasting chylomicronemia (MIM 144650). Lipids Health Dis. 2007;6:23.

16. Peterfy M, Ben-Zeev O, Mao HZ, Weissglas-Volkov D, Aouizerat BE, Pullinger $\mathrm{CR}$, et al. Mutations in LMF1 cause combined lipase deficiency and severe hypertriglyceridemia. Nat Genet. 2007;39:1483-7.

17. Cullen P. Evidence that triglycerides are an independent coronary heart disease risk factor. Am J Cardiol. 2000;86:943-9.

18. Athyros VG, Giouleme OI, Nikolaidis NL, Vasiliadis TV, Bouloukos VI, Kontopoulos AG, et al. Long-term follow-up of patients with acute hypertriglyceridemia-induced pancreatitis. J Clin Gastroenterol. 2002; 34:472-5

19. Hopkins PN, Wu LL, Hunt SC, Brinton EA. Plasma triglycerides and type III hyperlipidemia are independently associated with premature familial coronary artery disease. J Am Coll Cardiol. 2005;45:1003-12.

\section{Ready to submit your research? Choose BMC and benefit from:}

- fast, convenient online submission

- thorough peer review by experienced researchers in your field

- rapid publication on acceptance

- support for research data, including large and complex data types

- gold Open Access which fosters wider collaboration and increased citations - maximum visibility for your research: over $100 \mathrm{M}$ website views per year

At BMC, research is always in progress.

Learn more biomedcentral.com/submissions 